\title{
Reasons for cessation of clean intermittent catheterization after spinal cord injury: Results from the Neurogenic Bladder Research Group spinal cord injury registry
}

\author{
Darshan P. Patel MD ${ }^{1}$ Jennifer S. Herrick MS ${ }^{2}$ John T. Stoffel MD $^{3}$ | \\ Sean P. Elliott MD, MS ${ }^{4}$ Sara M. Lenherr MD ${ }^{1}$ Angela P. Presson PhD \\ Blayne Welk MD ${ }^{5}$ | Amitabh Jha MD, MPH ${ }^{6} \mid$ Jeremy B. Myers MD $^{1} \mid$ for the \\ Neurogenic Bladder Research Group (NBRG.org)
}

${ }^{1}$ Division of Urology, Department of Surgery, University of Utah, Salt Lake City, Utah

${ }^{2}$ Division of Epidemiology and Biostatistics, Department of Internal Medicine, University of Utah, Salt Lake City, Utah

${ }^{3}$ Department of Urology, University of Michigan, Ann Arbor, Michigan

${ }^{4}$ Department of Urology, University of Minnesota, Minneapolis, Minnesota

${ }^{5}$ Department of Surgery, University of Western Ontario, London, Ontario, Canada

${ }^{6}$ Department of Physical Medicine and Rehabilitation, University of Utah, Salt Lake City, Utah

\section{Correspondence}

Darshan P. Patel, MD, Division of Urology, Department of Surgery, University of Utah, 30 N 1900 E, RM\# 3B420, Salt Lake City, UT 84132. Email: Darshan.patel@hsc.utah.edu

Funding information

Patient-Centered Outcomes Research Institute, Grant/Award Number: CER14092138

\begin{abstract}
Introduction: Clean intermittent catheterization (CIC) is recommended for bladder management after spinal cord injury (SCI) since it has the lowest complication rate. However, transitions from CIC to other less optimal strategies, such as indwelling catheters (IDCs) are common. In individuals with SCI who stopped CIC, we sought to determine how individual characteristics affect the bladder-related quality of life (QoL) and the reasons for CIC cessation.

Methods: The Neurogenic Bladder Research Group registry is an observational study, evaluating neurogenic bladder-related QoL after SCI. From 1479 participants, those using IDC or urinary conduit were asked if they had ever performed CIC, for how long, and why they stopped CIC. Multivariable regression, among participants discontinuing CIC, established associations between demographics, injury characteristics, and SCI complications with bladder-related QoL.

Results: There were 176 participants who had discontinued CIC; 66 (38\%) were paraplegic and 110 (63\%) were male. The most common reasons for CIC cessation among all participants were inconvenience, urinary leakage, and too many urine infections. Paraplegic participants who discontinued CIC had higher mean age, better fine motor scores, and lower educational attainment and employment. Multivariable regression revealed years since SCI was associated with worse bladder symptoms (neurogenic bladder symptom score), $\geq 4$ urinary tract infections (UTIs) in a year was associated with worse satisfaction and feelings about bladder symptoms (SCI-QoL difficulties), while tetraplegia was associated better satisfaction and feelings about bladder symptoms (SCI-QoL difficulties).
\end{abstract}


Conclusions: Tetraplegics who have discontinued CIC have an improved QoL compared with paraplegics. SCI individuals who have discontinued CIC and have recurrent UTIs have worse QoL.

\section{K E Y W O R D S}

catheters, indwelling, intermittent urethral catheterization, patient-reported outcome measures, quality of life, spinal cord injuries, urinary bladder

\section{INTRODUCTION}

After spinal cord injury (SCI), most individuals develop neurogenic lower urinary tract dysfunction, which in the majority of cases requires assisted bladder emptying rather than volitional voiding. For most individuals with $\mathrm{SCI}$, this assisted bladder emptying includes catheterbased management, such as an indwelling catheter (IDC) or clean intermittent catheterization (CIC). IDCs, either a suprapubic tube (SPT) or Foley catheter, are associated with more urologic and nonurologic complications, including urinary tract infection (UTI), bladder and kidney stones, renal insufficiency, hydronephrosis, increased risk of bladder cancer, pressure ulcers, and allcause hospitalizations. ${ }^{1-4}$ Therefore, most clinicians and guidelines recommend CIC as the preferred bladder management strategy in individuals with SCI who require catheter-based management. ${ }^{5-7}$ However, despite the increased risk of complications associated with IDCs, up to $70 \%$ of individuals with SCI who use CIC after their injury transition to IDCs over time. ${ }^{8}$

The common reasons for management transitions from CIC to IDC have not been thoroughly investigated. Factors such as age, female sex, obesity, and upper extremity motor impairment are known to affect the ability to perform CIC. ${ }^{8-10}$ Clinical complications such as persistent urinary leakage, urethral erosion/stricture, perceived increased UTI risk, and autonomic dysreflexia have also been cited as reasons for management transitions. ${ }^{11}$ Recently, inconvenience with the frequency and time required to catheterize and dislike of the treatment modality has been associated with treatment transitions. ${ }^{12,13}$ However, other than these small studies, there is very little that is known about patient-reported factors associated with dissatisfaction with CIC and the quality of life (QoL) among individuals that have discontinued CIC. Crescenze et $\mathrm{al}^{14}$ showed that female sex, earlier injury, recurrent UTIs, and severe bowel dysfunction were associated with dissatisfaction and worse QoL among SCI individuals currently performing CIC. There are no similar studies regarding factors associated with dissatisfaction among SCI individuals who have discontinued CIC.
We examined the Neurogenic Bladder Research Group (NBRG) prospective registry of SCI participants to better understand factors associated with discontinuation and dissatisfaction with CIC. We hypothesize that individuals with SCI who have discontinued SCI with recurrent UTIs, severe bowel dysfunction, and chronic pain have worse QoL. In addition, tetraplegic individuals who have discontinued CIC are likely to have an improved QoL compared with paraplegics who have discontinued CIC.

\section{\begin{tabular}{l|l}
2 & METHODS
\end{tabular}}

The NBRG registry is a prospective observational study examining neurogenic-bladder-related QoL among individuals after SCI. The study was conducted at the Universities of Michigan, Minnesota, and Utah, and participants were recruited throughout the United States and Canada. Participants were allowed to enroll in the study through a telephonic interview and by answering electronic questionnaires. The study protocol and methods have been previously published. ${ }^{15}$ Eligible participants included those with an acquired SCI who had discontinued CIC, and were also $\geq 18$ years of age and spoke English. Individuals with progressive neurologic disorders (ie, multiple sclerosis) and congenital spinal cord malformations or neurologic injury (ie, cerebral palsy, myelomeningocele, etc) were excluded.

Our analysis cohort consisted of subjects who had discontinued CIC. Participants were asked about their current and past bladder management strategies during enrollment. Participants that reported IDC (SPT or Foley catheter) or urinary conduit (ileal or colon conduit) as their primary bladder management were asked if they had ever performed CIC. If the participants indicated they had done CIC in the past, they were asked about the timing of CIC cessation, categorized as duration of CIC for less than 1, 1 to 12 , and greater than 12 months. We gathered information about the participants from their enrollment interview, including (a) demographics and comorbidities: age, sex, education (bachelor's degree or higher), employment (employed for wages including 
self-employed), and Charlson Comorbidity Index ${ }^{16}$, (b) injury specifics: time from injury, injury level (either tetraplegic [cervical level 1-8] or paraplegic [thoracic, sacral, cauda equina]), and fine motor function, and (c) SCI complications: number of UTIs in the last year (selfreported rate as $0,1-3, \geq 4$ ), hospitalization for UTI in the last year, chronic pain (do you experience chronic pain?), as well as severe bowel dysfunction (neurogenic bowel dysfunction score $>14$ ). The neurogenic bowel dysfunction score is a validated self-reported measure of bowel dysfunction. ${ }^{17}$

Self-reported reasons for CIC discontinuation were gathered during enrollment interview. Participants were asked to select from a list of reasons for CIC discontinuation and multiple responses could be selected (Table 3). Participants were not asked to rank the reasons for CIC discontinuation. We stratified this data by the duration of CIC before cessation, sex, and level of injury.

\subsection{Outcome measures}

Fine motor function was measured using the SCI-QoL measurement system fine motor functioning subdomain that has been validated in individuals with SCI. ${ }^{18,19}$ This item bank has a calibrated mean score of 50 with score ranges between 0 and 100. A lower score conveys worse fine motor function. Symptoms and QoL were assessed using the neurogenic bladder symptom score (NBSS) and the SCI-QoL bladder management difficulties item bank. ${ }^{18,20}$ The NBSS focuses on bladder-related symptoms and complications with a score range between 0 and 74 , with lower scores indicating fewer symptoms. ${ }^{20}$ The NBSS also assesses satisfaction with the urinary system with a single question: "if you had to live the rest of your life with the way your bladder (or urinary reservoir) currently works, how would you feel?" (range, 0-4). ${ }^{20}$ The SCI-QoL difficulties item bank assesses feelings about bladder function and urinary incontinence. ${ }^{18}$ This item bank has a calibrated mean score of 50 with score ranges between 0 and 100. A lower score indicates fewer bladder difficulties.

\section{2 | Statistical methods}

Descriptive statistics of subjects who discontinued CIC were stratified by injury level (paraplegic and tetraplegic) including: means, standard deviations, medians, and interquartile ranges for the continuous variables and counts and column percentages for the categorical and ordinal variables were calculated. Comparisons with injury level for continuous variables were made using $t$ tests for the normally distributed variables and the Wilcoxon signed-rank test for the nonnormally distributed variables and categorical variables using $\chi^{2}$ tests. Among the full cohort of SCI individuals who discontinued CIC, univariable and multivariable linear regression models were created using a prespecified list of covariates that we hypothesized a priori. These models were used to determine whether the QoL outcome measures (NBSS total, NBSS satisfaction, and SCI-QOL bladder management difficulties) were significantly associated with demographic, injury characteristics, or injury-related complications among SCI individuals who discontinued SCI. Multivariable regression models were adjusted for duration of CIC, level of injury, age, sex, years since SCI, education, employment, severe bowel dysfunction, chronic pain, number of patient-perceived UTIs, and hospitalization for UTIs. Coefficients, 99.6\% confidence intervals (CIs), and $P$ values were reported from the models. We adjusted the $P$ values and $95 \%$ CIs within each model for multiple comparisons using the Bonferroni correction. Statistical analyses were conducted using the SAS software, version 9.4, SAS Institute Inc, Cary, NC.

\section{3 | RESULTS}

There were 1479 participants who enrolled in this study. Of these participants, 309 (20.8\%) identified their primary management as an IDC or a urinary conduit. In these participants, 176 (56.9\%) had used CIC in the past and discontinued this management for SPT $(113,64 \%)$, Foley catheter $(52,29.5 \%)$, or urinary conduit $(12,6.8 \%)$. Baseline characteristics of these participants are shown in Table 1 stratified by level of injury. In the unadjusted comparison between paraplegic and tetraplegic individuals who had discontinued CIC, paraplegic participants who had discontinued CIC had an older mean age and a larger proportion had a Charlson Comorbidity Index greater than 1 compared with tetraplegic participants. A significantly lower proportion of paraplegic participants reported a bachelor's degree or higher or employment compared with tetraplegic participants. Paraplegic participants had higher (better) fine motor scores compared with tetraplegic participants. Paraplegic participants also performed CIC a longer duration before cessation.

A higher proportion of paraplegic participants who discontinued CIC reported chronic pain. Overall, $16 \%$ of the cohort reported hospitalization for a UTI in the last year. There was no difference in the number of patientperceived UTI's and hospitalization for UTI when stratified by injury level.

The mean NBSS total score, NBSS satisfaction score, and SCI-QoL difficulties scores were improved in 
TABLE 1 Characteristics of spinal cord injury participants who discontinued clean intermittent catheterization

\begin{tabular}{|c|c|c|c|c|}
\hline Variable & Total $(\mathrm{n}=176)$ & Paraplegia $(n=66,38 \%)$ & Tetraplegia $(n=110,62 \%)$ & $P$ value ${ }^{*}$ \\
\hline Sex, male, n (\%) & $110(63 \%)$ & $39(59 \%)$ & $71(65 \%)$ & .47 \\
\hline Education, bachelor's degree or higher, n (\%) & $57(32 \%)$ & $10(15 \%)$ & $47(43 \%)$ & $<.001$ \\
\hline Employment, n (\%) $\omega$ & $41(23 \%)$ & $9(14 \%)$ & $32(29 \%)$ & .02 \\
\hline \multicolumn{5}{|l|}{ Duration of CIC before cessation, $\mathrm{n}(\%)$, mo } \\
\hline$<1$ & $20(11.4 \%)$ & $6(9.1 \%)$ & $14(12.7 \%)$ & .01 \\
\hline $1-12$ & $54(30.7 \%)$ & $12(18.2 \%)$ & $42(38.2 \%)$ & \\
\hline$>12$ & $102(58.0 \%)$ & $48(72.7 \%)$ & $54(49.1 \%)$ & \\
\hline \multicolumn{5}{|l|}{ Current bladder management } \\
\hline \multicolumn{5}{|l|}{ Indwelling catheter, n (\%) } \\
\hline SPT & $113(64.2 \%)$ & $38(57.6 \%)$ & $75(68.2 \%)$ & .36 \\
\hline Foley & $52(29.5 \%)$ & $23(34.8 \%)$ & $29(26.4 \%)$ & \\
\hline Urinary conduit & $12(6.8 \%)$ & $5(7.6 \%)$ & $7(6.4 \%)$ & .76 \\
\hline \multicolumn{5}{|l|}{ Injury-related complications } \\
\hline \multicolumn{5}{|l|}{ Number of UTIs, n (\%) } \\
\hline 0 & $37(21 \%)$ & $17(26 \%)$ & $20(18 \%)$ & .30 \\
\hline $1-3$ & $79(45 \%)$ & $25(38 \%)$ & $54(50 \%)$ & \\
\hline Median (IQR) & $17.0(11,25)$ & $20.5(15,28)$ & $15.0(10,23)$ & \\
\hline NBSS satisfaction $\phi$-mean (SD) & $2.0(1.3)$ & $2.3(1.3)$ & $1.8(1.3)$ & .01 \\
\hline Median (IQR) & $2.0(1.0,3.0)$ & $2.0(1.0,4.0)$ & $2.0(1.0,2.0)$ & \\
\hline SCI-QoL difficulties $T$ score-mean (SD) & $57.0(8.1)$ & $59.8(8.7)$ & $55.3(7.2)$ & $<.001$ \\
\hline Median (IQR) & $57.5(52.6,61.6)$ & $59.5(57.5,64.8)$ & $57.0(50.9,59.9)$ & \\
\hline
\end{tabular}

Note: $\omega$ employment-making wages (including self-employment), $\delta$ chronic pain—participants asked "do you experience chronic pain?" and $\gamma$ severe bowel dysfunction-neurogenic bowel dysfunction score $\geq 14$. $\phi$ NBSS satisfaction is the final question of the NBSS "If you had to live your life with the way your bladder (or urinary reservoir) currently works, how would you feel?"

Missing values: SCI-FI fine motor $T$ score $=2$, severe bowel dysfunction $=25$, hospitalization for UTI $=1$, and SCI-QOL bladder management difficulties $T$ score $=2$.

Abbreviations: CIC, clean intermittent catheterization; IDC, indwelling catheter; IQR, interquartile range; NBSS, neurogenic bladder symptom score; QoL, quality of life; SCI, spinal cord injury; SCI-QoL, spinal cord injury measurement system; SCI-QoL difficulties, bladder management difficulties item bank; SPT, suprapubic tube; UTI, urinary tract infection.

${ }^{*}$ Analysis of variance or $\chi^{2}$ test as appropriate.

tetraplegic participants who discontinued CIC compared with paraplegic participants (Table 1).

The reasons for CIC cessation among the 176 participants in our cohort are shown in Table 2. Among the entire cohort, convenience $(36 \%)$, urinary leakage
(20\%), and the number of urinary infections (19\%) were the most common reasons for CIC cessation. The most common reasons were similar when stratified by sex. When responses were stratified by injury level, tetraplegic participants reported "I did not want to be 


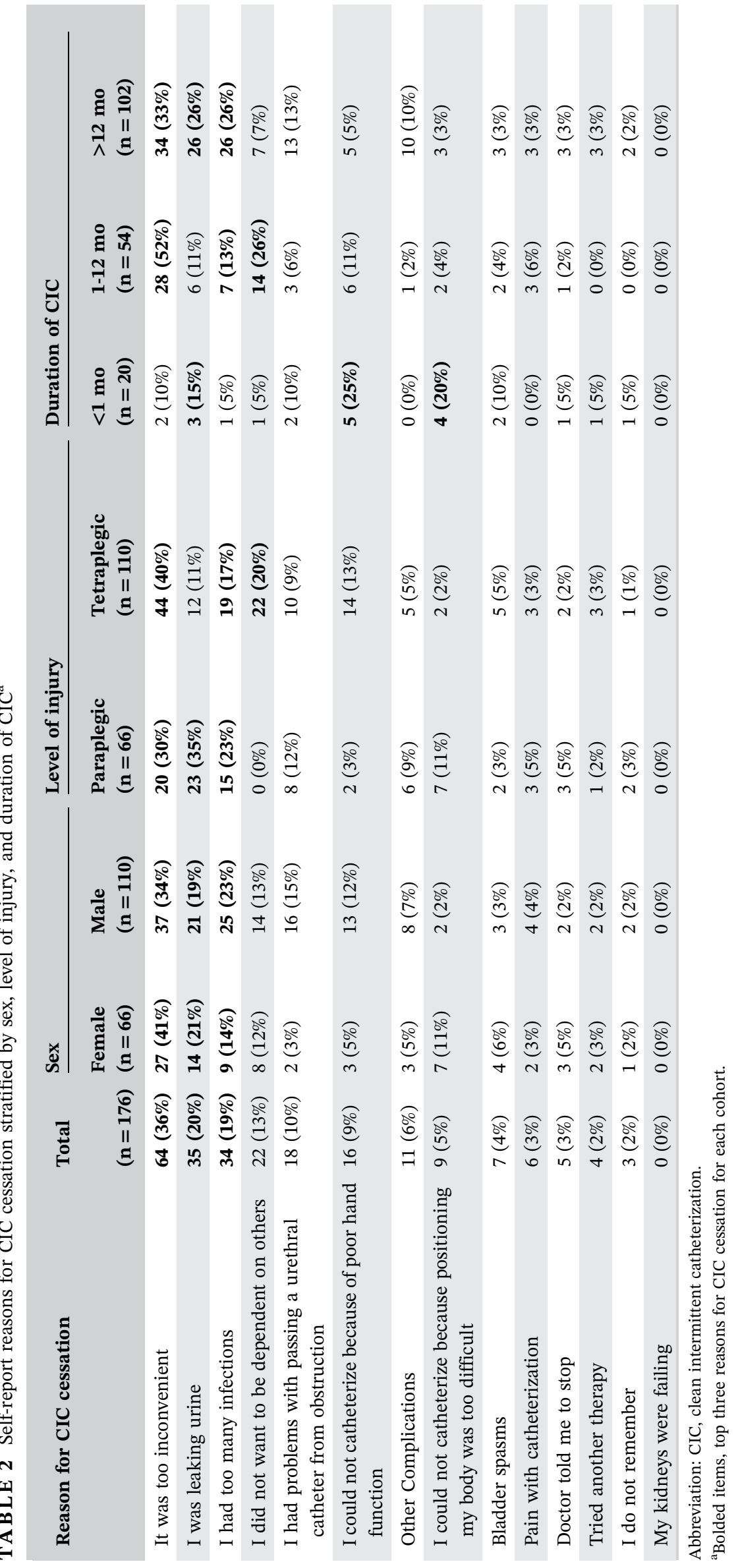


T A B LE 3 Multivariate regression ${ }^{\text {a }}$ of effects of demographics, injury characteristics, and injury-related complications on NBSS total score, NBSS satisfaction, and SCI-QoL difficulties $T$ score

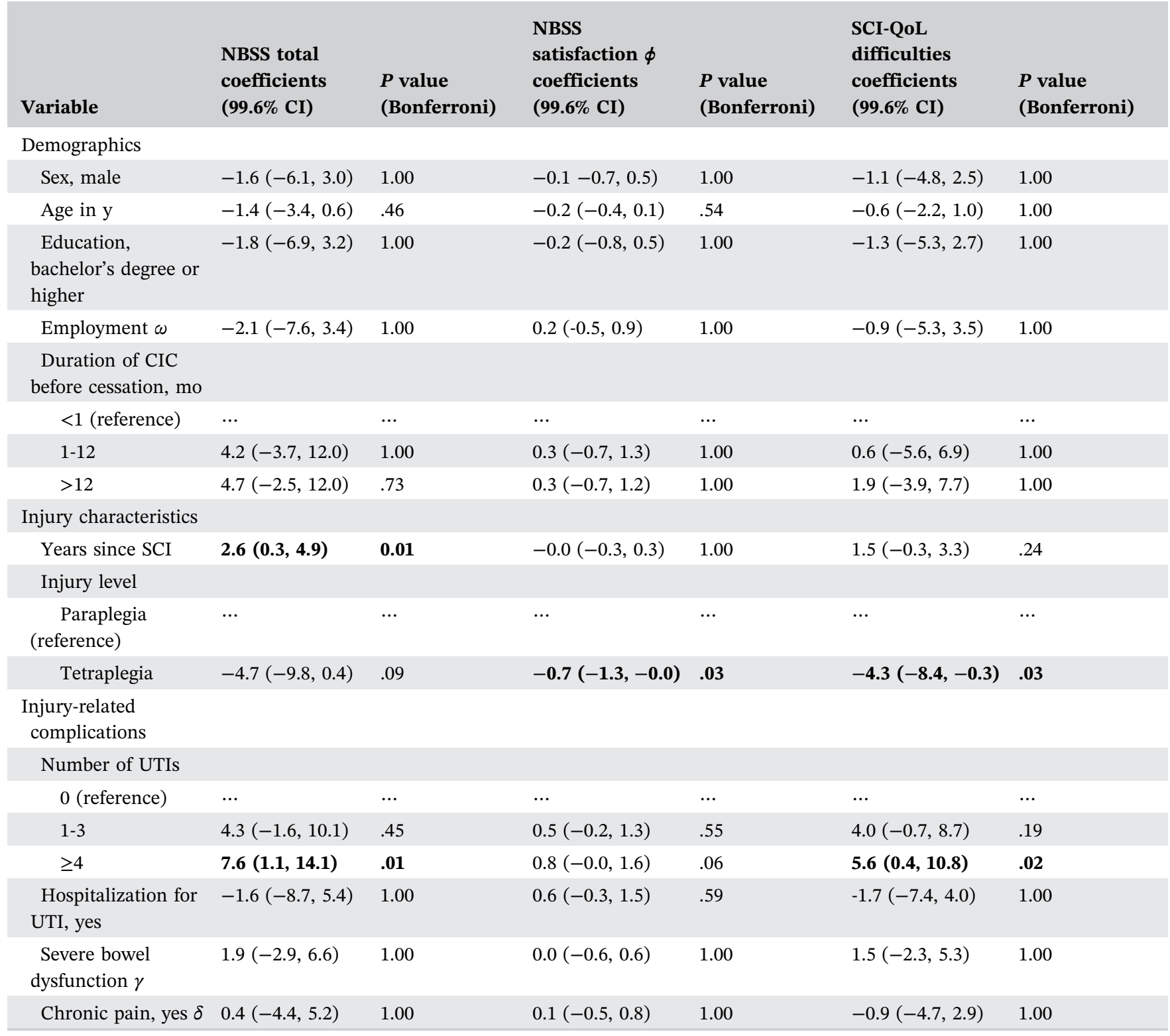

Note: $\omega$ employment—making wages (including self-employment), $\gamma$ severe bowel dysfunction—neurogenic bowel dysfunction score $\geq 14$, and $\delta$ chronic pain participants were asked "do you experience chronic pain?" $\phi$ NBSS satisfaction is the final question of the NBSS "If you had to live your life with the way your bladder (or urinary reservoir) currently works, how would you feel?"

Abbreviations: CIC, clean intermittent catheterization; NBSS, neurogenic bladder symptom score; QoL, quality of life; SCI, spinal cord injury; SCI-QoL, spinal cord injury measurement system; SCI-QoL difficulties, bladder management difficulties item bank; UTI, urinary tract infection.

${ }^{a}$ Adjusting for the duration of CIC, level of injury, age, sex, years since SCI, education, employment, severe bowel dysfunction, chronic pain, number of UTIs, and hospitalization for UTIs.

dependent on others" as the second most common reason for CIC cessation compared with none of the paraplegic respondents. In participants who had discontinued CIC within 1 month of initiation, poor hand function, and body positioning were more common reasons compared with longer duration of CIC before discontinuation.

Multivariate linear regression models examining the associations between demographics and comorbidities, injury characteristics, and SCI complications on the mean NBSS total score, NBSS satisfaction score, and SCIQoL difficulties scores are shown in Table 3. Among SCI participants who had discontinued CIC, years from SCI were associated with worse NBSS total scores. In addition, participants who discontinued CIC who reported $\geq 4$ patient-perceived UTI within the last year, had significantly worse NBSS total scores and SCI-QoL difficulties scores. Tetraplegic participants had improved NBSS satisfaction and SCI-QoL difficulties scores. 


\section{4 | DISCUSSION}

Catheter-based management is common after SCI. However, despite the established higher clinical complications associated with certain strategies such as IDC, there is a little that is known about the characteristics of individuals with SCI who transition to IDC or urinary conduit from CIC or the reasons they make this decision. We examined the NBRG prospective registry to better understand reasons for CIC discontinuation and factors associated with dissatisfaction among those SCI participants who have discontinued CIC. The top three selfreported reasons for CIC cessation for the entire cohort were inconvenience, urinary leakage, and too many urine infections. We found that despite lower fine motor scores among tetraplegic participants that discontinued CIC, these participants had improved bladder-related QoL when compared with paraplegic participants who discontinued CIC. Participants who discontinued CIC and had recurrent UTIs ( $\geq 4$ patient-perceived UTI within the last year) had worse bladder-related QoL. Severe bowel dysfunction and chronic pain were not associated with changes in bladder-related QoL among SCI participants who discontinued CIC.

Prior studies evaluating the reasons for treatment transition among individuals with SCI have been limited in their number and scope. Afsar et $\mathrm{al}^{11}$ explored reasons for discontinuation of CIC in a retrospective review of recently injured individuals with SCI at a single institution. At discharge from rehabilitation, 104 (64\%) individuals were performing CIC, while subsequent follow-up showed that only 60 (37.5\%) of individuals continued CIC and $9(21 \%)$ had transitioned from CIC to IDC for bladder management. The authors reported recurrent UTI, urinary incontinence, urolithiasis, the dependence of caregivers, and urethral strictures as reasons for discontinuation of CIC. Age, sex, education, and level of injury were not associated with compliance with CIC. In another study, 248 members of the Norwegian Spinal Cord Injuries Association were surveyed, and the leading reasons given for bladder management other than CIC included: "it suits me best" $(24 \%)$ and "it gives me flexibility with more options" $(21 \%){ }^{21}$ When stratified by injury level (paraplegic vs tetraplegic), the authors found a significant difference in responses, with tetraplegic individuals reporting "it gives me flexibility with more options," "it does nt constrain my daily activities," "it is less time consuming," and "it suits me best" more often than paraplegic participants. Responses were not mutually exclusive and were not stratified by injury level.

More recently, Lane et $\mathrm{al}^{12}$ presented their survey results of 100 veterans with prior traumatic SCI at the Minneapolis Veterans Affairs Medical Center. They found that 22 of the 46 participants (48\%) using IDC had prior history of CIC which had been discontinued. The authors found that the most common reasons for the transition from CIC were inconvenience $(n=5)$, clinician recommendation $(n=5)$, and dislike of CIC $(n=4)$. Clinician recommendation was an unexpected reason for treatment transition given the well-established goals in SCI care of maintaining CIC when possible and may suggest knowledge gaps about the higher complications associated with IDCs.

Zlatev et $\mathrm{al}^{10}$ previously demonstrated that impairment in upper extremity motor function is an important predictor of long-term CIC adoption. For patients that performed CIC for shorter durations before discontinuation, patient-reported reasons of "I could not catheterize because of poor hand function" and "I could not catheterize because positioning my body was too difficult" are reflective. However, overall, the most common reasons for CIC discontinuation included inconvenience, urinary incontinence, and recurrent UTI. This suggests poor participant understanding of clinical complications associated with various bladder management strategies, as it is well known that IDC have the highest relative rate of urinary complications including recurrent UTI. ${ }^{1,22}$ These self-reported reasons from a large cohort of SCI participants who discontinued CIC may be helpful in designing interventions to improve long-term CIC adoption.

Interestingly, we also found that tetraplegic participants who discontinued CIC had lower (improved) NBSS satisfaction and SCI-QoL difficulties scores compared with paraplegic participants, although this association was relatively small. Myers et $\mathrm{al}^{23}$ show similar findings in another analysis performed using this registry. The authors found that both tetraplegic and paraplegic participants using IDC as primary bladder management strategy had lower (improved) NBSS total scores. However, tetraplegic participants reporting IDC as primary bladder management strategy had significantly lower (improved) SCI-QoL difficulties scores. This association was not significant among paraplegic participants using IDC. Overall QoL among tetraplegic participants using CIC as the primary bladder management strategy is likely related to improvement in urinary concerns. ${ }^{24} \mathrm{In}$ a study of SCI participants performing CIC alone, CIC with botulinum toxin, and CIC with augmentation cystoplasty, tetraplegia had lower (improved) NBSS total and SCIQoL difficulties. ${ }^{25}$ Conversely, there was no association between the level of injury and fine motor hand function on dissatisfaction among participants reporting CIC as a bladder management strategy, when patients who underwent any procedure to improve urinary-related QoL were excluded. ${ }^{14}$ 
There are several notable limitations to this study. One limitation is that this study uses participant-reported data based on their perception of their health, which had not been corroborated with clinical data or chart review, such as information regarding urinary infections. Also, we did not ask participants to rank the most important reasons for discontinuation of CIC, which limits our analysis. We chose to stratify our findings based on injury level (paraplegia vs tetraplegia) in the multivariable analysis as a proxy for upper extremity motor function rather than use fine motor scores as we felt there would be too much collinearity. Inclusion bias is also a common limitation of survey studies; those participants with bladder problems are more likely to enter the study in hopes of learning more about their bladder. Finally, there is potential for recall errors for specific reported variables, such as duration of CIC before discontinuation, which may impact our findings.

However, this study is strengthened by a large diverse study population and extensive yet robust data about demographics, injury characteristics, SCI complications, and patient-reported outcomes. Understanding how these characteristics influence QoL in individuals who transition their bladder management from CIC, as well as the reasons that individuals decide to transition their management, is paramount to developing shared decision-making tools to individualize the optimal bladder management strategy for SCI. Decision aids may avoid management transitions to less optimal bladder strategies by educating, addressing individuals concerns, and potential QoL issues with transitioning management. Discrepancies exist between clinical and patient-reported outcomes for bladder management among individuals with SCI; however, a careful balance between avoiding complications and the best bladder-related QoL is essential.

\section{5 | CONCLUSION}

In this analysis of the NBRG prospective registry, we found that the most common self-reported reasons for CIC cessation among individuals with SCI were inconvenience, urinary leakage, and too many urine infections. Participants with tetraplegia who had discontinued CIC had greater satisfaction with their urinary system and less impact of bladder symptoms in their QoL compared with participants with paraplegia. Participants who discontinued CIC and had recurrent UTIs ( $\geq 4$ patient-perceived UTI within the last year) had worse bladder-related QoL. An individualized approach aimed at balancing clinical complications with QoL concerns is needed when exploring treatment transitions for SCI bladder management.

\section{ACKNOWLEDGMENTS}

This study was supported through a Patient-Centered Outcomes Research Institute (PCORI) Award (CER14092138). All statements in this report, including its findings and conclusions, are solely those of the authors and do not necessarily represent the views of PCORI. The following study is dedicated to Jason Hall, an advocate for the study of SCI care, dedicated father, husband, and inspiration to all those that met him (1971-2019).

\section{ORCID}

John T. Stoffel (1) http://orcid.org/0000-0002-5503-4940 Blayne Welk (D) http://orcid.org/0000-0001-7093-558X Jeremy B. Myers (D) http://orcid.org/0000-0002-5786-1552

\section{REFERENCES}

1. Weld KJ, Dmochowski RR. Effect of bladder management on urological complications in spinal cord injured patients. $J$ Urol. 2000;163(3):768-772.

2. Bennett CJ, Young MN, Adkins RH, Diaz F. Comparison of bladder management complication outcomes in female spinal cord injury patients. J Urol. 1995;153(5):1458-1460.

3. Groah SL, Weitzenkamp DA, Lammertse DP, Whiteneck GG, Lezotte DC, Hamman RF. Excess risk of bladder cancer in spinal cord injury: evidence for an association between indwelling catheter use and bladder cancer. Arch Phys Med Rehabil. 2002;83(3):346-351.

4. Chen Y, DeVivo M, Roseman J. Current trend and risk factors for kidney stones in persons with spinal cord injury: a longitudinal study. Spinal Cord. 2000;38(6):346-353.

5. Jaggi A, Drake M, Siddiqui E, Fatoye F. A comparison of the treatment recommendations for neurogenic lower urinary tract dysfunction in the national institute for health and care excellence, European Association of Urology and international consultations on incontinence guidelines. Neurourol Urodyn. 2018;37:2273-2280.

6. Drake MJ, Apostolidis A, Cocci A, et al. Neurogenic lower urinary tract dysfunction: clinical management recommendations of the Neurologic Incontinence committee of the Fifth International Consultation on Incontinence 2013. Neurourol Urodyn. 2016;35(6):657-665.

7. Groen J, Pannek J, Castro Diaz D, et al. Summary of European Association of Urology (EAU) Guidelines on Neuro-Urology. Eur Urol. 2016;69(2):324-333.

8. Cameron AP, Wallner LP, Tate DG, Sarma AV, Rodriguez GM, Clemens JQ. Bladder management after spinal cord injury in the United States 1972 to 2005. J Urol. 2010;184(1):213-217.

9. Yavuzer G, Gök H, Tuncer S, Soygür T, Arikan N, Arasil T. Compliance with bladder management in spinal cord injury patients. Spinal Cord. 2000;38(12):762-765.

10. Zlatev DV, Shem K, Elliott CS. Predictors of long-term bladder management in spinal cord injury patients-upper extremity function may matter most. Neurourol Urodyn. 2018;37(3): 1106-1112. 
11. Afsar SI, Yemisci OU, Cosar SNS, Cetin N. Compliance with clean intermittent catheterization in spinal cord injury patients: a long-term follow-up study. Spinal Cord. 2013;51(8):645-649.

12. Lane GI, Driscoll A, Tawfik K, Chrouser K. A cross-sectional study of the catheter management of neurogenic bladder after traumatic spinal cord injury. Neurourol Urodyn. 2018;37(1): 360-367.

13. Vaidyanathan S, Soni BM, Singh G, Oo T, Hughes PL. Barriers to implementing intermittent catheterisation in spinal cord injury patients in Northwest Regional Spinal Injuries Centre, Southport, U.K. ScientificWorldJournal. 2011;11:77-85.

14. Crescenze IM, Myers JB, Lenherr SM, et al. Predictors of low urinary quality of life in spinal cord injury patients on clean intermittent catheterization. Neurourol. Urodyn. 2019;38:13321338.

15. Patel DP, Lenherr SM, Stoffel JT, et al. Study protocol: patient reported outcomes for bladder management strategies in spinal cord injury. BMC Urol. 2017;17(1):95.

16. Charlson ME, Pompei P, Ales KL, MacKenzie CR. A new method of classifying prognostic comorbidity in longitudinal studies: development and validation. $J$ Chronic Dis. 1987;40(5):373-383.

17. Krogh K, Christensen P, Sabroe S, Laurberg S. Neurogenic bowel dysfunction score. Spinal Cord. 2006;44(10):625-631.

18. Tulsky DS, Kisala PA, Tate DG, Spungen AM, Kirshblum SC. Development and psychometric characteristics of the SCI-QOL bladder management difficulties and bowel management difficulties item banks and short forms and the SCI-QOL bladder complications scale. J Spinal Cord Med. 2015;38(3): 288-302.

19. Tulsky DS, Kisala PA, Victorson D, et al. Overview of the spinal cord injury-quality of life (SCI-QOL) measurement system. $J$ Spinal Cord Med. 2015;38(3):257-269.
20. Welk B, Morrow S, Madarasz W, Baverstock R, Macnab J, Sequeira K. The validity and reliability of the neurogenic bladder symptom score. J Urol. 2014;192(2):452-457.

21. Hagen EM, Rekand T. Management of bladder dysfunction and satisfaction of life after spinal cord injury in Norway. J Spinal Cord Med. 2014;37(3):310-316.

22. Roth JD, Pariser JJ, Stoffel JT, et al. Patient subjective assessment of urinary tract infection frequency and severity is associated with bladder management method in spinal cord injury. Spinal Cord. 2019;57:700-707.

23. Myers JB, Lenherr SM, Stoffel JT, et al. Patient reported bladder related symptoms and quality of life after spinal cord injury with different bladder management strategies. $J$ Urol. 2019;202:574-584. https://doi.org/101097ju0000000000000270

24. Patel DP, Elliott SP, Stoffel JT, Brant WO, Hotaling JM, Myers JB. Patient reported outcomes measures in neurogenic bladder and bowel: a systematic review of the current literature. Neurourol Urodyn. 2016;35(1):8-14.

25. Myers JB, Lenherr SM, Stoffel JT, et al. The effects of augmentation cystoplasty and botulinum toxin injection on patient-reported bladder function and quality of life among individuals with spinal cord injury performing clean intermittent catheterization. Neurourol Urodyn. 2019;38(1):285-294.

How to cite this article: Patel DP, Herrick JS, Stoffel JT, et al. Reasons for cessation of clean intermittent catheterization after spinal cord injury: Results from the Neurogenic Bladder Research Group spinal cord injury registry. Neurourology and Urodynamics. 2020;39:211-219. https://doi.org/10.1002/nau.24172 